\title{
Le varianti A1 e A2 della $\beta$-caseina non hanno un effetto significativo sul microbiota del latte
}

\author{
The A1 and A2 $\beta$-casein variants have not impact on milk microbiota
}

\author{
${ }^{1}$ Consiglio Nazionale delle Ricerche, Istituto di Biologia e Biotecnologia Agraria, Lodi, Italy \\ 2Università degli studi di Parma, Dipartimento di Scienze Medico Veterinarie, Parma, Italy \\ ¿Università Cattolica del Sacro Cuore, Dipartimento di Scienze animali, Alimentazione e Nutrizione, Facoltà di Agraria, Scienze Alimentari e Ambientali, Piacenza, Italy \\ ${ }^{4}$ Consiglio Nazionale delle Ricerche, Istituto di Scienze delle Produzioni Alimentari, Milano, Italy
}

\section{*Corresponding author:}

Bianca Castiglioni

IBBA-CNR, Via Einstein s/n, I-26900, Lodi.

Tel. 0371-4662504 Email: bianca.castiglioni@ibba.cnr.it
Ricevuto il 21 aprile 2020

Accettato il 19 maggio 2020

DOI: 10.36138/STLC.03.2020.02

\section{Riassunto}

Obiettivo: Negli ultimi anni il latte prodotto da animali omozigoti per la variante $\mathrm{A} 2$ della $\beta$-caseina, una delle lattoproteine, è stato ritenuto più digeribile $\mathrm{e}$ salutare di quello $\mathrm{A} 1$ e pertanto ne è aumentata la commercializzazione, dapprima solo in paesi quali Australia, Nuova Zelanda, Stati Uniti e Cina, successivamente anche in Europa e in Italia. Lo scopo del presente lavoro è stato quello di verificare se le varianti A1 e A2 della $\beta$-caseina potessero influenzare anche il microbiota del latte.

Materiali e metodi: Trenta bovine di razza Frisona Italiana sono state suddivise in tre gruppi di dieci animali ciascuno in base al loro genotipo al locus della $\beta$-caseina. Sui campioni di latte individuale, di latte di massa e formaggio a pasta molle filata raccolti da ciascun gruppo sperimentale in due diverse stagioni (inverno/estate) è stata effettuata un'analisi metagenomica basata sul sequenziamento massivo (Next Generation
Sequencing) del gene 16S rDNA. Risultati: L'analisi tassonomica ha mostrato che le varianti A1 e A2 della $\beta$-caseina non influiscono in maniera significativa né sulla composizione del microbioma del latte né su quello del formaggio molle a pasta filata. Differenze significative sono invece state osservate tra i campioni raccolti nelle due stagioni con prevalenza dei phyla Firmicutes in inverno e Proteobacteria in estate.

Conclusioni: Questi risultati preliminari hanno rivelato che, l'ambiente influenza maggiormente la composizione del microbiota del latte rispetto al genotipo degli animali.
Parole chiave:
latte
formaggio
- $\beta$-caseina
variante $\mathrm{A} 2$
microbioma

\begin{abstract} complex microbiota which can be influenced by numerous factors such as the season, the diet, the type of litter, the facility. As well, the animal's genotype can affect the composition of the milk microbiota. In milk, $\beta$-casein is characterized by a high-level of polymorphism. The aim of this study was to verify if $A 1$ and A2 $\beta$-casein variants could influence the milk microbioma. Materials and methods: Thirty Holstein cows were genotyped for $\beta$-casein locus and divided into three groups. In two different seasons, milk samples (individual and bulk tank for each experimental group) and mozzarella cheese were collected separately from each group. Bacterial DNA was extracted from milk and cheese samples and analysed by
\end{abstract}

Objective: Milk is composed by a using metagenomic approach. 


\section{INTRODUZIONE}

Studi recenti hanno rilevato che anche la ghiandola mammaria, al pari di altri organi come ad esempio il rumine, ospita una variegata e complessa comunità microbica, la cui composizione può influire sia sulla qualità del latte che sulla salute dell'animale (1). La composizione del microbiota del latte può essere influenzata da numerosi fattori per lo più ambientali, come ad esempio la temperatura, l'umidità, la dieta, l'ambiente di stabulazione, la routine di mungitura (2-6). Anche il genotipo dell'animale può avere una influenza sulla composizione del microbiota del latte, come illustrato recentemente nel lavoro di Cremonesi e collaboratori (7), che descrive come il microbiota del latte di bovine appartenenti a due razze diverse, la Frisona Italiana e la Rendena, allevate nella stessa stalla e nelle stesse condizioni ambientali, presenti differenze che potrebbero incidere sia sulla salute della ghiandola mammaria che sulle caratteristiche dei prodotti lattiero-caseari.

Tra le proteine del latte, la $\beta$-caseina rappresenta circa il 35\% delle caseine nel latte bovino, e presenta il più alto grado di polimorfismo tra le caseine (8). È noto come i polimorfismi delle lattoproteine possano influenzare le proprietà casearie e biologiche del latte (9). Ad oggi sono state scoperte diverse varianti genetiche della $\beta$-caseina: A1, A2, A3, B, C, D, E, F, G, H1, H2, e I (10). Tra queste, le varianti A1 e $\mathrm{A} 2$ sono le più diffuse nelle bovine da latte, sia nella razza Frisona che Bruna, la maggior parte delle quali è eterozigote $A 1 A 2$.

Le varianti A1 e A2 differiscono per la presenza nella posizione 67 della catena amminoacidica dell'istidina (nel caso di A1) e della prolina (nel caso di A2). Da un punto di vista biologico ciò comporta una minore digeribilità della $\beta$-caseina $A 1$ rispetto alla $A 2(11)$. In conseguenza di ciò, soprattutto in Australia, Nuova Zelanda, Stati Uniti e Cina, ma anche in Europa e in Italia, è aumentata la commercializzazione di prodotti, latte e yogurt soprattutto, a base di latte prodotto da vacche omozigoti A2A2. Dal punto di vista biologico, si ritiene che tale effetto sia legato al rilascio del peptide BCM7 (beta-casomorfina 7) che viene rilasciato durante i processi digestivi soprattutto dalla variante $\mathrm{A} 1$ e molto meno dalla $\mathrm{A} 2(8,12-14)$. Si tratta di un oppioide naturale che può avere un effetto negativo sulla salute umana qualora venga assorbito (8). Poco si è indagato invece dei possibili effetti delle varianti della $\beta$-caseina a livello caseario (15). In realtà, alcune ricerche hanno osservato il rilascio della $\mathrm{BCM} 7$ anche durante la digestione del latte $\mathrm{A} 2$, seppur in quantità molto più modeste (12-14).

Pertanto, lo scopo del presente studio è stato quello di valutare l'effetto delle varianti A1 e A2 della $\beta$-caseina bovina sulla composizione del microbioma del latte e dei prodotti caseari.

\section{MATERIALI E METODI}

\section{Selezione degli animali e disegno sperimentale}

Sono state condotte due prove sperimentali presso l'Azienda Agroecologica Sperimentale di Carpaneta (MN) in due stagioni differenti: inverno (marzo 2019) ed estate (luglio 2019). Per tutte le vacche in lattazione erano disponibili i genotipi al locus della $\beta$-caseina. In ciascuna prova sperimentale, sono stati formati 3 gruppi sperimentali, composti da circa 10 vacche ciascuno, in rapporto al genotipo degli animali: $A 1 A 1, A 1 A 2$ e A2A2. In entrambe le prove sperimentali, $i$ gruppi sperimentali A1A2 e A2A2 erano composti da 10 vacche. Le vacche di genotipi $A 1 A 1$ sono state 8 nella prova di marzo e 7 in quella di luglio. Nell'ambito di ciascuna prova, le vacche dei tre gruppi erano paragonabili tra loro per giorni in lattazione, numero di lattazione e per livello produttivo. Alle bovine era stata somministrata una razione a base di insilati ( $48 \%$ della sostanza secca della razione). La sostanza secca (33\%) era composta per il $14,7 \%$ di proteina grezza, il $4,7 \%$ di estratto etereo, il $31,5 \%$ di NDF e il $44,4 \%$ di NFC. L'ingestione giornaliera di sostanza secca era mediamente di $25,4 \mathrm{~kg}$. La media delle cellule somatiche (SCC) nel latte di massa destinato alla trasformazione nel mese di marzo era di $192 \times 10^{3}$ per il genotipo $A 1 A 1,47 \times 10^{3}$ per il genotipo $A 2 A 2$ e $198 \times 10^{3}$ per il genotipo $A 1 A 2$, mentre nel mese di luglio 44, 58, e $146 \times 10^{3}$ rispettivamente per i genotipi $A 1 A 1, A 2 A 2$ e A1A2. Nel mese di marzo la carica batterica standard (CBS) del latte aveva un valore di 6,92 + 0,87 Log10 ufc/L per il genotipo $A 1 A 1,6,24+0,34$ per il genotipo $A 1 A 2$ e 6,50 + 0,71 per il genotipo $A 2 A 2$, mentre nel mese di luglio i valori di CBS erano $6,16+0,88$ Log10 ufc/L, 6,23 + 0,55 e 6,29 + 0,43, rispettivamente per i genotipi A1A1, A1A2 e A2A2. Durante ogni prova sperimentale sono stati raccolti campioni (i) di latte individuale come pool dei quattro quarti, (ii) di latte di massa per ogni gruppo sperimentale (pool vacche/gruppo), e (iii) di formaggi tipo mozzarella ottenuti dalla lavorazione del latte di massa di ogni gruppo sperimentale in due caseificazioni successive. La caseificazione a mozzarella non ha previsto l'impiego di starter ed è stata ottenuta con aggiunta di acido citrico. I campioni raccolti sono stati immediatamente congelati a $-20^{\circ} \mathrm{C}$ e trasferiti in laboratorio per le analisi molecolari.

\section{Estrazione del DNA, librerie e analisi metagenomica}

Dai 55 campioni di latte individuale, 12 di latte di massa di ogni gruppo sperimentale, 12 di formaggio tipo mozzarella è stato estratto il DNA batterico secondo i protocolli descritti in letteratura a partire da latte (16) e da formaggio (17). Successivamente, le regioni variabili V3-V4 del gene che codifica per la subunità 16S dell'RNA ribosomiale sono state amplificate seguendo il protocollo standard per la 
Tabella I. Composizione tassonomica a livello di phylum del latte individuale, latte di massa e mozzarella (\% delle abbondanze relative) dei tre differenti genotipi (A1A1, A1A2, A2A2)

Table I - Taxonomic composition at phylum level of individual milk, bulk tank milk and mozzarella cheese samples (\% of relative abundances) for the three different genotypes (A1A1, A1A2, A2A2)

\begin{tabular}{|c|c|c|c|c|c|}
\hline & & \multicolumn{3}{|c|}{ GENOTIPO } & \\
\hline & & A1A1 & A1A2 & A2A2 & \\
\hline Phylum & Campione & $\%$ & $\%$ & $\%$ & p.value \\
\hline \multirow[t]{3}{*}{ Firmicutes } & Latte individuale & 49.7 & 54.5 & 52.4 & 0.8043237 \\
\hline & Latte di massa & 39.4 & 46.4 & 48.6 & 0.8269292 \\
\hline & Mozzarella & 15.1 & 8.5 & 15.6 & 0.8096516 \\
\hline \multirow[t]{3}{*}{ Proteobacteria } & Latte individuale & 17.9 & 13.0 & 17.3 & 0.3317903 \\
\hline & Latte di massa & 40.6 & 34.5 & 39.2 & 0.8610986 \\
\hline & Mozzarella & 72.3 & 85.2 & 80.2 & 0.5319503 \\
\hline \multirow[t]{3}{*}{ Bacteroidetes } & Latte individuale & 17.5 & 16.1 & 13.8 & 0.2757905 \\
\hline & Latte di massa & 17.3 & 16.2 & 10.2 & 0.6005026 \\
\hline & Mozzarella & 11.7 & 5.1 & 3.5 & 0.4459083 \\
\hline \multirow[t]{3}{*}{ Actinobacteria } & Latte individuale & 10.4 & 11.0 & 10.4 & 0.8482379 \\
\hline & Latte di massa & 1.4 & 1.5 & 1.0 & 0.8329565 \\
\hline & Mozzarella & 0.4 & 0.9 & 0.4 & 0.7131132 \\
\hline \multirow[t]{3}{*}{ Others } & Latte individuale & 4.5 & 5.4 & 6.1 & - \\
\hline & Latte di massa & 1.2 & 1.4 & 1.0 & - \\
\hline & Mozzarella & 0.5 & 0.3 & 0.3 & - \\
\hline
\end{tabular}

preparazione delle librerie Illumina e sequenziate sulla piattaforma Miseq (Illumina, San Diego, USA). Dopo aver verificato la qualità dei dati ottenuti, l'analisi bioinformatica è stata eseguita mediante la pipeline QIIME 1.9 (18) e l'assegnazione tassonomica è stata effettuata utilizzando database SILVA 123 (https://www.arb-silva.de/).

\section{RISULTATI E DISCUSSIONE}

Come mostrato in Figura 1 ogni tipologia di campione analizzato (latte individuale, latte di massa e mozzarelle ottenuti da ognuno dei tre gruppi sperimentali) presenta una propria diversità batterica (Fig. 1A). A livello di phylum la composizione del microbioma del latte individuale è costituita prevalentemente da Firmicutes (52\%), Proteobacteria (16\%), Bacteroidetes (15\%) e Actinobacteria (11\%), così come nel latte di massa (Firmicutes $45 \%$, Proteobacteria $38 \%$, Bacteriodetes $15 \%$ e Actinobacteria 1\%), mentre quella della moz- zarella prevalentemente da Proteobacteria (79\%), seguiti da Firmicutes (13\%) e Bacteroidetes (circa 7\%). Tale diversità è confermata anche dall'analisi della beta-diversity (Fig. 1B), analisi applicata per mostrare quanto è diversa la composizione microbica in un ambiente rispetto ad un altro, basandosi sulla differenza nei profili di abbondanza tassonomica dei diversi campioni. Le differenze significative trovate tra il microbioma del latte e quello delle mozzarelle sono inoltre in accordo con quanto descritto in precedenza sia da Ercolini e collaboratori (19) che da Mauriello e collaboratori (20). Durante la produzione di mozzarella, avvenuta utilizzando acido citrico e non con l'impiego di starter, la temperatura di filatura abbinata all'acidificazione della cagliata portano alla selezione di un microbiota tipico composto prevalentemente da batteri lattici termofili.

Analizzando i campioni in base ai diversi genotipi, come riportato nella Tabella I, il microbioma del latte individuale a livello di phylum è caratterizzato prevalentemente da Firmicutes, seguito da Proteo- 
bacteria, Bacteroidetes e Actinobacteria, a costituire oltre il 95\% della componente microbica presente nel campione. Nel latte di massa i due phyla predominanti sono risultati essere Firmicutes e Proteobacteria per i tre genotipi analizzati; inoltre nella mozzarella i Proteobacteria rappresentano anche il phylum maggiormente rap- presentato per tutti i genotipi analizzati. Dall'analisi della varianza delle abbondanze relative dei phyla si evince che le differenze fra i vari genotipi al locus della $\beta$-caseina non influiscono in maniera significativa sulla composizione del microbioma del latte e dei formaggi molli a pasta filata.

Figura 1. Analisi tassonomica (1A) e beta-diversity (1B) dei campioni di latte individuale, del latte di massa e della mozzarella a livello di phylum

Figure 1. Distribution of the sequence relative abundances summarized at phylum level $(1 A)$ and beta-diversity (1B) for individual milk, bulk tank milk and mozzarella cheese.
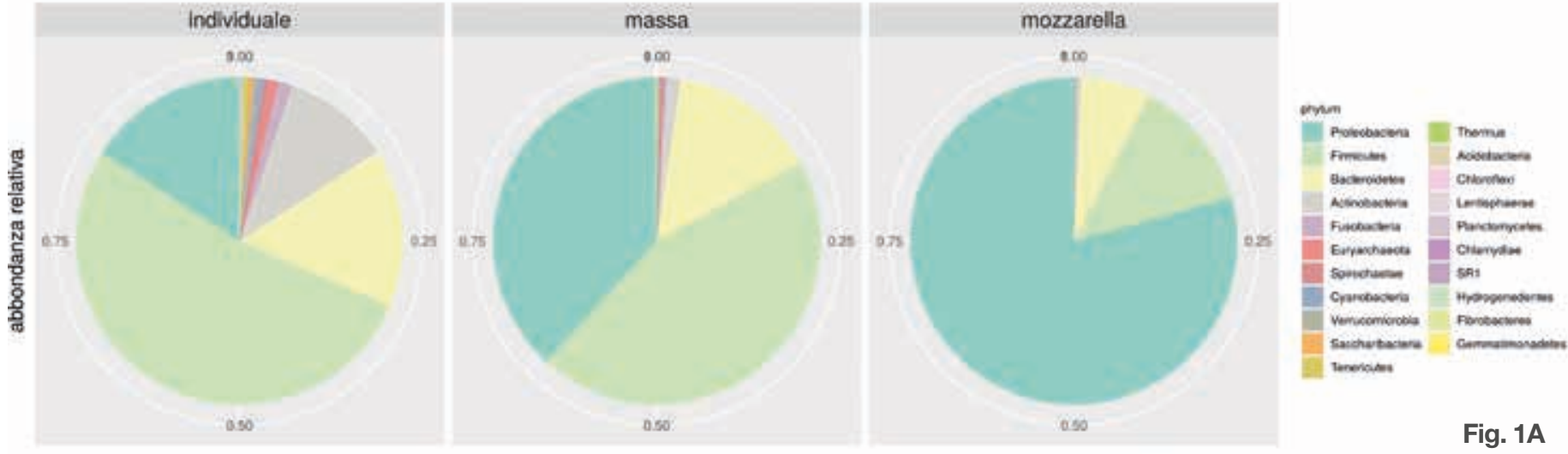

Fig. 1A
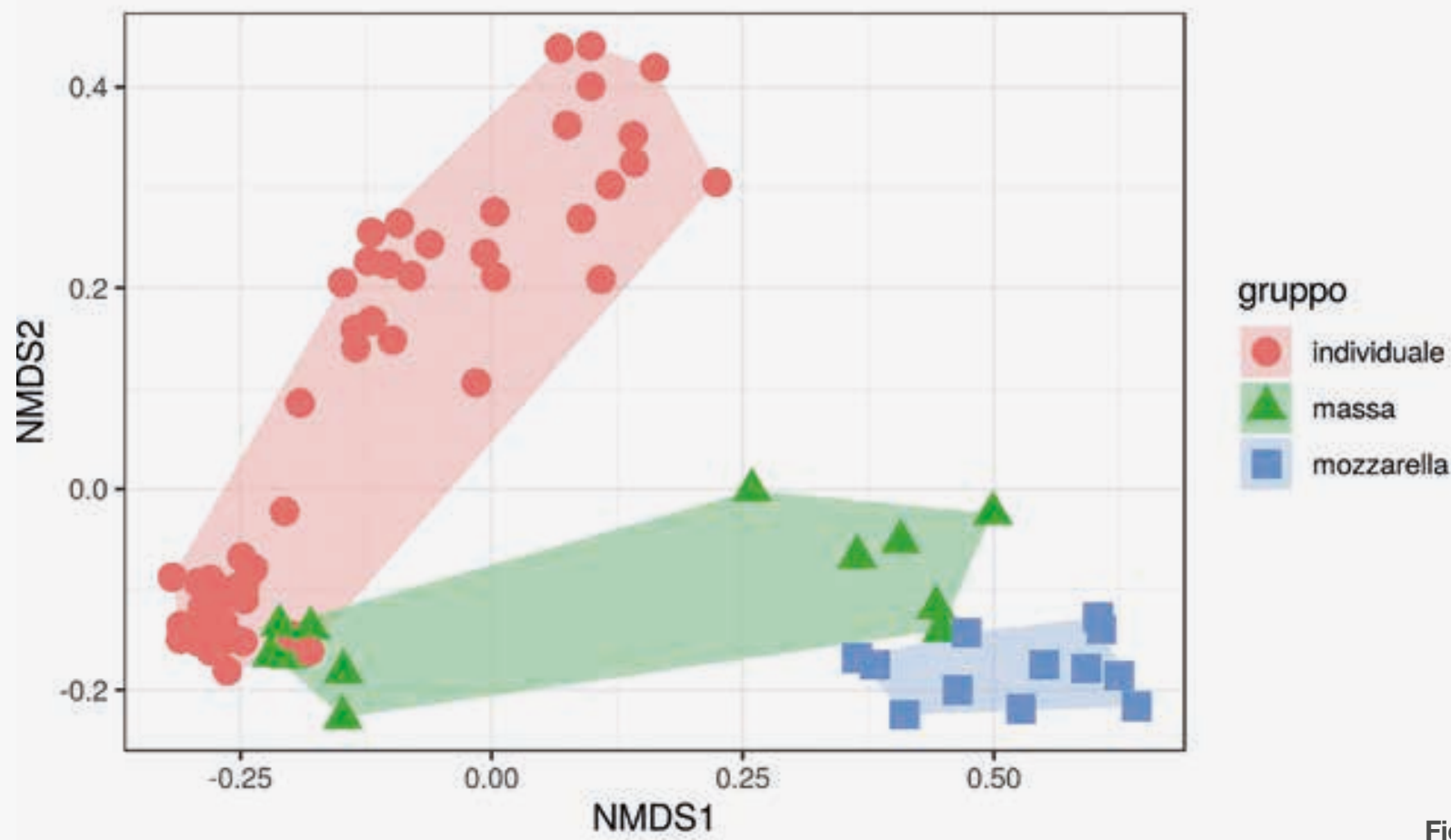
Figura 2. Analisi tassonomica dei campioni a livello di phylum (2A) e betadiversity (2B) nelle due stagioni considerate (inverno = marzo; estate = luglio)

Figure 2. Distribution of the sequence relative abundances summarized at phylum level $(2 A)$ and beta-diversity (2B) for the two different seasons (winter = March; summer = July)
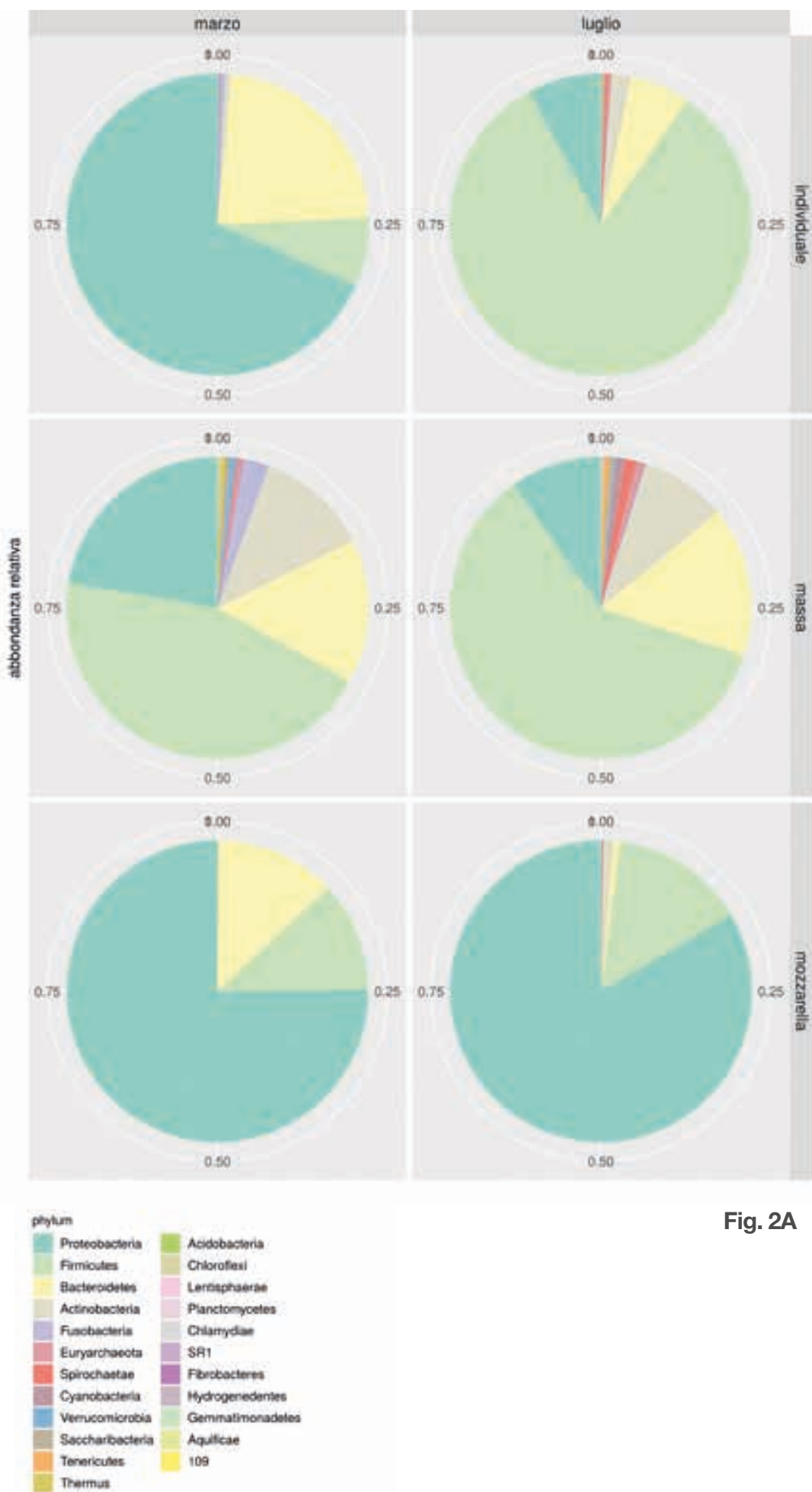

Le abbondanze relative a livello di phylum sono risultate invece variare in modo significativo nelle due stagioni di campionamento considerate: infatti, come mostrato nella Figura $2 \mathrm{~A}$, nel periodo invernale la composizione del microbioma di latte e mozzarella per i tre differenti genotipi è costituita prevalentemente da Firmicutes per il $60 \%$ circa, seguita da Proteobacteria per il $20 \%$, Bacteroidetes per il $12 \%$ e Actinobacteria per il $7 \%$ circa, mentre nella stagione estiva è costituita prevalentemente da Proteobacteria per il 37\%, da Firmicutes per il 34\%, da Bacteroidetes per il $16 \%$ e da Actinobacteria per il $9 \%$ circa. Anche l'analisi della beta-diversity evidenzia come la composizione del microbiota sia più influenzata dalla stagione in cui è stato effettuato il campionamento piuttosto che dal genotipo degli animali (Fig. 2B). Risultati simili sono stati recentemente riportati nel lavoro di Li e collaboratori nel 2018 (21) che evidenzia come tali cambiamenti siano determinati dalle condizioni climatiche, soprattutto dalla temperatura e dall'umidità relativa.

\section{CONCLUSIONI}

In conclusione, i risultati ottenuti in questo studio mostrano che la composizione del microbioma del latte e dei formaggi molli a pasta filata risulta essere più influenzata dai fattori ambientali stagionali piuttosto che dal genotipo al locus della $\beta$-caseina degli animali.

\section{CONFLITTO DI INTERESSE}

Gli autori dichiarano che non esistono conflitti di interesse economico da parte di uno o più autori.

\section{RINGRAZIAMENTI}

Questa ricerca è stata finanziata dal progetto AGER 2 "FARM-INN" grant 2017-1130.

\section{BIBLIOGRAFIA}

1. Young W., Hine B.C., Wallace O.A., Callaghan M., Bibiloni R. Transfer of intestinal bacterial components to mammary secretions in the cow. PeerJ. 2015; 3:e888.

Fig. $2 A \quad$ 2. Kuehn J.S., Gorden P.J., Munro D., Rong R., Dong Q., Plummer P.J., et al. Bacterial community profiling of milk samples as a means to understand culture-negative bovine clinical mastitis. PLoS One. 2013; 8(4):e61959.

3. Oikonomou G., Bicalho M.L., Meira E., Rossi R.E., Foditsch C., Machado V.S., et al. Microbiota of cow's milk; distinguishing healthy, sub-clinically and clinically diseased quarters. PLoS One. 2014. 9, e85904.

4. Zhang R., Huo W., Zhu W., Mao S. Characterization of bacterial community of raw milk from dairy cows during subacute ruminal acidosis challenge by high-throughput sequencing. J. Sci. Food Agric. 2015 95, 1072-1079. 

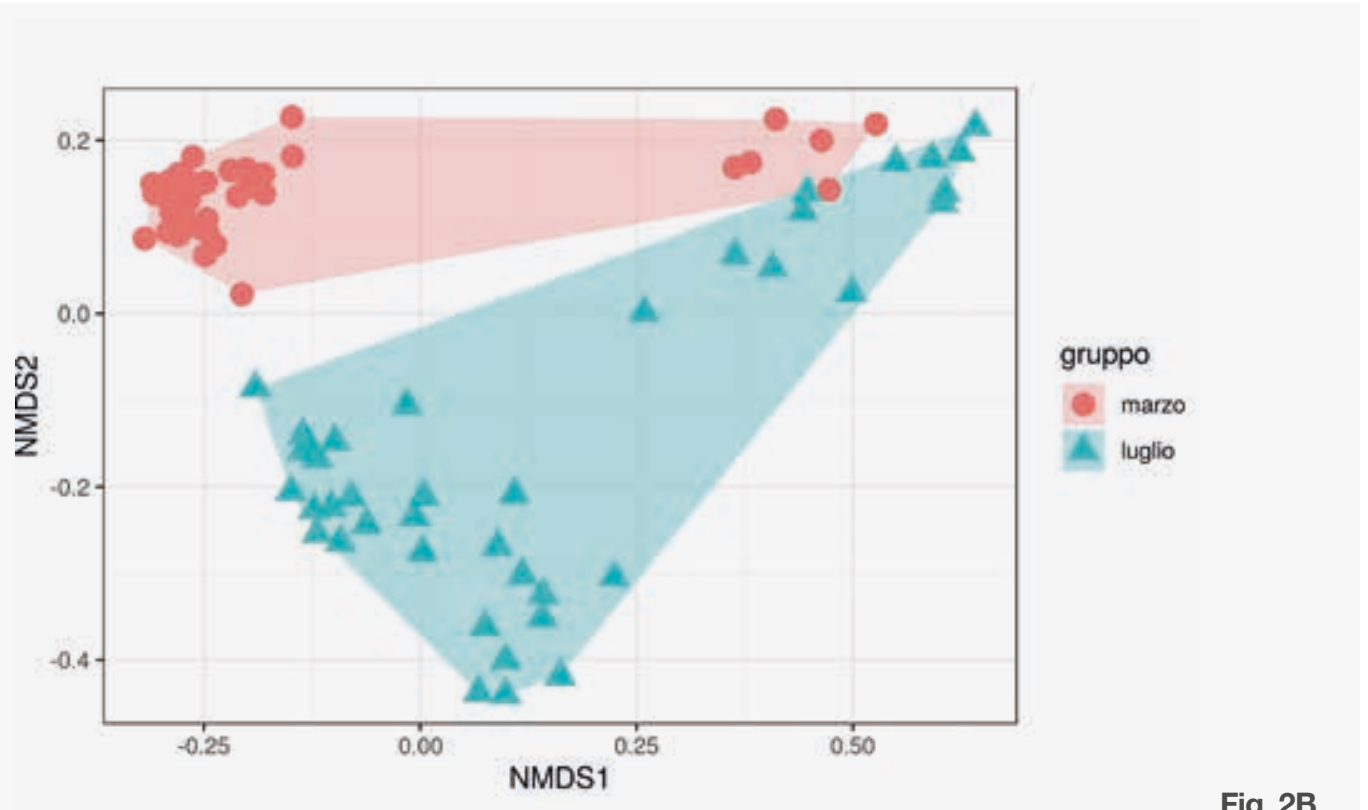

Fig. 2B

5. Falentin H., Rault L., Nicolas A., Bouchard D.S., Lassalas J., Lamberton P., et al. Bovine Teat Microbiome Analysis Revealed Reduced Alpha Diversity and Significant Changes in Taxonomic Profiles in Quarters with a History of Mastitis. Front. Microbiol. 2016, 7, 480.

6. Derakhshani H., Fehr K.B., Sepehri S., Francoz D., DeBuck J., Barkema H.W., et al. Invited review: microbiota of the bovine udder: contributing factors and potential implications for udder health and mastitis susceptibility. J. Dairy Sci. 2018. 101,10605-10625.

7. Cremonesi P., Conte G., Severgnini M., Turri F., Monni A., Capra E., et al. Evaluation of the effects of different diets on microbiome diversity and fatty acid composition of rumen liquor in dairy goat. Animal. 2018. 12(9):1856-1866.

8. Summer A., Di Frangia F., Ajmone Marsan P., De Noni I., Malacarne M. Occurrence, biological properties and potential effects on human health of $\beta$-casomorphin 7: Current knowledge and concerns. Crit. Rev. Food Sci. Nutr. 2020. 1-19.

9. Caroli A.M., Chessa S., Erhardt G.J. Invited review: milk protein polymorphisms in cattle: effect on animal breeding and human nutrition. J. Dairy Sci. 2009. 92(11):5335-5352.

10. Huppertz T. 2013. Chemistry of caseins. In Advanced Dairy Chemistry (Volume 1A), ed. P.L.H McSweeney and P.F. Fox, 135-160. 4th ed. Boston; Springer Science.

11. EFSA (European Food Safety Authority). Review of the potential health impact of b-casomorphins and related peptides. EFSA Scientific Report. 2009. 231:1-107.

12. Asledottir T., Le T.T., Thu T.T., Petrat-Melin B., Devold T.G., Larsen L.B., et al. Identification of bioactive peptides and quantification of b-casomorphin-7 from bovine b-casein A1, A2 and I after ex vivo gastrointestinal digestion. Int. Dairy J. 2017. 71: 98-106.

13. Asledottir T., Le T.T., Poulsen N.A., Lotte T.G., Larsen B., Vegarud G.E. Rele- ase of b-casomorphin-7 from bovine milk of different b-casein variants after ex vivo gastrointestinal digestion. Int. Dairy J. 2018. 81: 8-11.

14. Cieslinska A., Kostyra E.B., Kostyra H., Olenski K., Fiedorowicz E., Kaminski S.A. Milk from cows of different $\beta$-casein genotypes as a source of $\beta$-casomorphin-7. Int. J. Food Sci. Nutr. 2012. 63:426-430.

15. Poulsen N.A., Rosengaard A.K., Szekeres B.D., Gregersen V.R., Jensen H.B. and Larsen L.B. Protein heterogeneity of bovine $\beta$-casein in Danish dairy breeds and association of rare $\beta$-casein $F$ with milk coagulation properties. ACTA Agr. Scan. - Animal Sci. 2016. 66: 190-198.

16. Cremonesi P., Castiglioni B., Malferrari G., Biunno I., Vimercati C., Moroni P., et al. Technical note: Improved method for rapid DNA extraction of mastitis pathogens directly from milk. J. Dairy Sci. 2006. 89(1): 163-169.

17. Silvetti T., Capra E., Morandi S., Cremonesi P., Decimo M., Gavazzi F., et al. Mirobial population profile during ripening of Protected Designation of Origin (PDO) Silter cheese, produced with and without autochthonous starter culture. LWT - Food Science and Technology. 2017. 84: 821-831

18. Caporaso J.G., Kuczynski J., Stombaugh J., Bittinger K., Bushman F.D., Costello E.K., et al. QIIME allows analysis of high-throughput community sequencing data. Nat. Methods. 2010. 7(5):335-336.

19. Ercolini D., De Filippis F., La Storia A., lacono M. "Remake" by high-throughput sequencing of the microbiota involved in the production of water Buffalo mozzarella cheese. Appl. Environ. Microbiol. 2012. 78, 8142-8145.

20. Mauriello G., Moio L., Genovese A., Ercolini D. Relationships between flavouring capabilities, bacterial composition and geographical origin of natural whey cultures used for traditional water-buffalo Mozzarella cheese manufacture. J. Dairy Sci. 2003. 86: 486-497.

21. Li N., Wang Y., You C., Ren J., Chen W., Zheng H., et al. Variation in Raw Milk Microbiota Throughout 12 Months and the Impact of Weather Conditions. Scientific Reports. 2018. 8:237 